\title{
Dorsal stream activation during retrieval of object size and shape
}

\author{
ROBYN T. OLIVER and SHARON L. THOMPSON-SCHILL \\ University of Pennsylvania, Philadelphia, Pennsylvania
}

\begin{abstract}
We investigated dorsal visual stream involvement in the retrieval of a variety of visual attributes of common objects, using functional magnetic resonance imaging. Seven subjects made binary decisions about the shape, color, and size of named objects during scanning. Bilateral parietal activity was significantly greater during retrieval of shape and size information than during retrieval of color information. Consistent with a domain-specific distributed model of semantic organization, the finding that dorsal stream activity is associated with size and shape retrieval, as compared with color retrieval, may indicate that both size and shape information are learned partly through dorsally mediated processes, such as visually guided grasping. These results demonstrate that both visual-processing streams (i.e., the ventral "what" pathway and the dorsal "where" pathway) are involved in the storage and/or retrieval of knowledge of object appearance but that, just as in vision, these two pathways may play different roles in conceptual processing.
\end{abstract}

We gather information about objects through daily interaction with our environment. The question of how this semantic information is organized in the brain is a matter of considerable debate. Although some theories of semantic memory describe representations as being abstracted from any particular input or output system (e.g., Smith \& Medin, 1981), other theories describe semantic information as a collection of distributed mental representations that are implemented in functionally and physically distinct systems that correspond to different sensorimotor domains (e.g., Allport, 1985; Warrington $\&$ Shallice, 1984). The latter class of theories will be referred to here as domain-specific, distributed models of semantic memory. Unlike unitary models of semantic representations, domain-specific distributed models make specific predictions about the relationship between how information is acquired and how (and where) that information is stored. In the present study, we test such a prediction within the domain of visual knowledge retrieval. Specifically, we used functional magnetic resonance imaging (fMRI) to examine whether our knowledge about the appearance of objects is distributed across distinct neural pathways involved in visual perception.

Research from the domains of visual perception (Ungerleider \& Mishkin, 1982) and visual semantic memory (Kellenbach, Brett, \& Patterson, 2001) has indicated that there may be reason to expect differences in the brain activity associated with the retrieval of different types of

This research was funded through NIH Grant MH60414. We thank Russell Epstein and Irene Kan for commenting on an earlier version of this manuscript. Correspondence concerning this article should be addressed to R. T. Oliver, Department of Psychology, University of Pennsylvania, 3815 Walnut Street, Philadelphia, PA 19104-6196 (e-mail: robyn@psych.upenn.edu). visual attributes. On the basis of numerous studies of the visual system in nonhuman primates, Ungerleider and Mishkin proposed that two visual information-processing streams flow forward from the primary visual cortex: the ventral visual-processing stream identifies objects from their visual features (i.e., "what"), whereas the dorsal stream processes their spatial locations (i.e., "where"). Neuropsychological evidence suggests that two routes for visual information processing are also found in humans and are involved in visual imagery tasks. For example, Levine, Warach, and Farah (1985) observed dissociations between imagery for visual and spatial information in patients with ventral and dorsal lesions, respectively. An alternative account of the dorsal versus ventral stream division has been offered by Goodale and Milner (1992), who argued that the key function of the dorsal stream is to mediate visually guided motor movements. This account of dorsal stream function has been supported by studies of patients with parietal lesions, who have difficulty forming the appropriate grasp size and orientation for objects, despite their ability to adequately describe the spatial location of these object (Jeannerod, 1988; Perenin \& Vighetto, 1988).

Thus, both Goodale and Milner (1992) and Ungerleider and Mishkin (1982) have argued for a functional dissociation between the dorsal and the ventral streams, although they disagree about the function of the dorsal stream. In both theories, however, the ventral stream is proposed to be involved in object identification. Numerous neuroimaging studies have supported the claim that visual semantic information relevant to object identification is stored in the ventral stream (Kellenbach et al., 2001; Lee et al., 2002; Martin, Haxby, Lalonde, Wiggs, \& Ungerleider, 1995; Thompson-Schill, Aguirre, D’Esposito, \& Farah, 1999; Vandenberghe, Price, Wise, Josephs, \& 
Frackowiak, 1996; for a review of this literature, see Thompson-Schill, 2003). For example, Chao and Martin (1999) compared the activity associated with retrieving the color of objects represented in grayscale object drawings with the activity associated with viewing grayscale Mondrian-type images; this comparison revealed activity in the left fusiform and lingual gyri and in the right lingual gyrus. Some authors (e.g., Kellenbach et al., 2001; Thompson-Schill et al., 1999) have pointed out that ventral activity associated with visual attribute retrieval, such as the fusiform activity for color retrieval described above (Chao \& Martin, 1999), is consistent with domain-specific distributed models of semantic memory (e.g., Allport, 1985).

If the structures involved in the processing of visual information are also involved in the forming or storing of long-term representations of that information, one might also expect semantic retrieval of at least some visual attributes to activate regions in the dorsal visual-processing stream. Consistent with this hypothesis, Kellenbach et al. (2001) found dorsal stream activity associated with the retrieval of object size. They argued that their results were consistent with the what/where model of the two visual streams. They hypothesized that size information about objects is acquired through spatial comparison between objects and that retrieval of this information should be associated with activity near the spatial-processing mechanism in the dorsal stream.

Although this prediction follows logically when the what/where model of dorsal stream function in considered in the context of the domain-specific distributed model of memory, one might also predict dorsal stream involvement in size retrieval when the what/how model of dorsal stream processing (Goodale \& Milner, 1992) is considered in the context of domain-specific distributed models. Goodale and Milner have argued that the dorsal visualprocessing stream serves to guide visually mediated actions, such as grasping. Because distributed models are concerned primarily with how information is acquired initially, it follows that if the activity of reaching is one way in which size information is learned and reaching is associated with dorsal structures, dorsal structures should be involved in the retrieval of size information. The same prediction would also be made for any other visual semantic attributes for which it is the case that a substantial part of its representation is learned through grasping or other behaviors mediated by the dorsal stream. In particular, if this hypothesis is correct, information about object shape may also involve the dorsal stream.

The aim of the present fMRI study was to test the involvement of the dorsal stream during size, shape, and color retrieval tasks. On the basis of Kellenbach et al.'s (2001) results, there was reason to believe that activity may be found in the dorsal stream for semantic retrieval of size information. However, previous studies in which semantic retrieval by attribute type has been tested have not included a shape retrieval condition for comparison with color or size retrieval. In the present study, we ex- amined the role of the posterior parietal cortex in the retrieval of a variety of visual attributes, in order to further elucidate the distributed nature of semantic representations and, potentially, to extend our knowledge about the function of the dorsal visual-processing stream.

\section{METHOD}

\section{Subjects}

Five males and 3 females participated in this fMRI study. However, 1 of the male subjects was excluded from analysis, due to excessive motion. The subjects ranged in age from 18 to 28 years, and all were right-handed native English speakers. They were also screened for history of psychiatric or neuropsychological illness and current use of drugs affecting the central nervous system. Participation consisted of a 15-min training session the day before the scan and a 2-h session, which included about $1.5 \mathrm{~h}$ of scan time. The subjects gave informed consent and were compensated $\$ 35$ for their participation.

\section{Materials}

Each subject completed four tasks: three semantic retrieval tasks and one nonsemantic (baseline) task. Each semantic retrieval task required the subject to make a judgment about a named object on the basis of a single visual attribute: shape, color, or size. The nonsemantic baseline task was lexical decision for abstract words and pronounceable nonwords. For each of the three semantic tasks, the subjects made judgments about 36 unique objects. Therefore, a total of 108 stimuli were used. During the shape task, the subjects were asked to judge whether the named object was composed of more curved edges or more straight edges. For the color task, the subjects were required to decide whether the named object was colored or not colored (e.g., black, white, gray, silver, or clear). In the size task, the subjects determined whether named objects were small or large (no object was provided as a reference). In order to select stimuli with high agreement, we conducted a survey of 20 volunteers (none of whom participated in the fMRI study). For each task condition, half of the items were selected because they elicited one of the two instructed responses (e.g., "large") in at least $80 \%$ of the survey subjects, whereas the other half of the items were selected because they elicited the alternative response (e.g., "small") in at least $80 \%$ of the survey subjects. Items were balanced across semantic task conditions for letter length, familiarity, word frequency (log transformed), imageability, and concreteness, as rated in the MRC Psycholinguistics Database (Wilson, 1987).

The nonsemantic (baseline) task was an auditory lexical decision task. The subjects heard 135 pronounceable letter strings (67 words and 68 nonwords) and were asked to determine whether each stimulus they heard was a word or a nonword. The words used in the lexical decision task were abstract words with MRC imageability and concreteness ratings of below 400. All the words used in the lexical decision task were matched to the semantic task on letter length, familiarity, and word frequency (log transformed), as rated in the MRC Psycholinguistics Database (Wilson, 1987).

Each semantic retrieval task was presented in a single run of an fMRI session, using an alternating blocked design. In each condition, blocks of nine experimental items alternated with blocks of nine lexical decision items (see Figure 1). A single block of nine practice trials was inserted before the blocked set of experimental and baseline conditions. Items were pseudorandomized into blocks so as to include roughly equal numbers of each type of response (e.g., colored vs. not colored) in each block of nine and so as to make the blocks roughly the same in terms of concreteness, familiarity, frequency, imageability, and letter length. Short instructions preceded each block of trials to remind the subject which task to perform. Each fMRI run included four experimental blocks (shape, color, or size) and five baseline blocks (lexical decision). Stimuli 


\section{Shape Task}

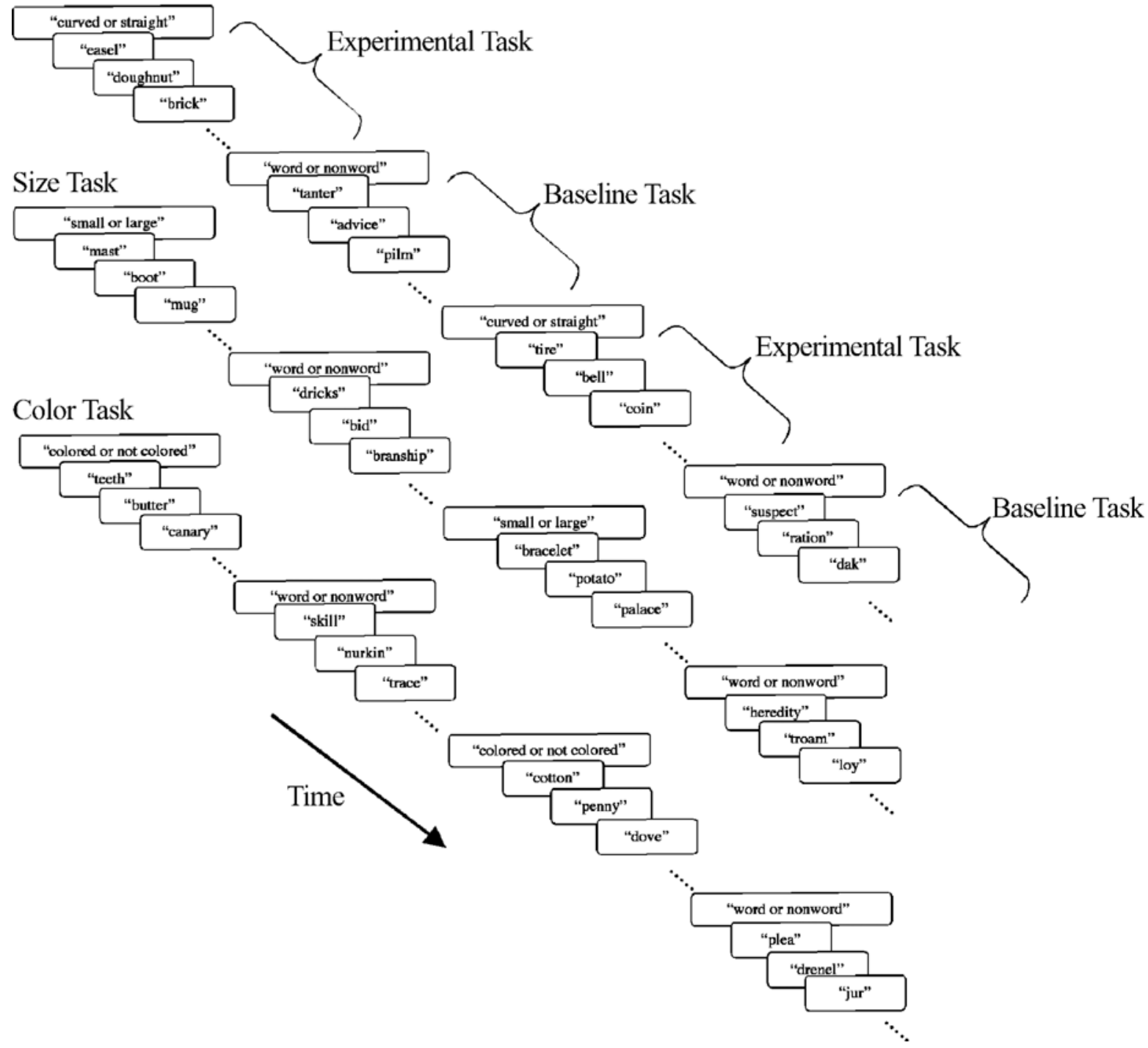

Figure 1. The structure of a typical experimental task run for each condition.

were presented every $2 \mathrm{sec}$, and each block of stimuli was $20 \mathrm{sec}$ long $(2 \mathrm{sec}$ of instructions and $18 \mathrm{sec}$ of stimuli). All materials were recorded in SoundEdit 16 (Version 2), using a female voice for all the stimuli and a male voice for all the instructional cues.

\section{Procedure}

Each subject visited the lab 1 or 2 days prior to the fMRI session in order to practice the tasks and complete paperwork. During the practice session, the subject was presented with a 63 -item practice set of nonexperimental items and was asked to perform a shortened version of each type of experimental condition. For each attribute task (size, shape, and color), two blocks of 4 items were alternated with two blocks of 4 lexical decision items.

The subjects were scanned in a 1.5-T GE Signa scanner (G.E. Medical Systems, Milwaukee, WI) using a standard head coil. Foam padding was used to reduce head motion within the coil. For each subject, T1-weighted anatomical scans were performed before functional images were acquired. Next, each of the three experimental conditions (shape, color, and size) was run in one of three orders. During each of the three experimental task runs, the blood oxygenation level dependent (BOLD) technique (Ogawa et al., 1993) was used to acquire 90 sets of 21 interleaved, axial gradient echo, echoplanar images at a rate of $2 \mathrm{sec}$ per acquisition $(\mathrm{TE}=50$, $64 \times 64$ pixels in a $24-\mathrm{cm}$ field of view, voxel size $=3.75 \times 3.75 \times$ $5 \mathrm{~mm}$ ). Each experimental scan lasted $3 \mathrm{~min} 20 \mathrm{sec}$. Stimuli were presented auditorily using PsyScope 1.2.5 software (Cohen, MacWhinney, Flatt, Provost, 1993) on a Macintosh G3 Powerbook in conjunction with Avotec's auditory and visual presentation system (Stuart, FL, www.avotec.org). To maintain synchrony between image acquisition and stimulus presentation, initiation of stimulus presentation was cued by a signal sent from the scanner. For all the tasks, the subjects were asked to respond as quickly and accurately as possible, using their thumbs on a four-button response pad. Stimuli in all conditions were presented every $2 \mathrm{sec}$. Responses were made by simultaneously pressing the inner two buttons to indicate one response (e.g., colored) and the outer two buttons to indicate the other response (e.g., not colored).

As was discussed above, each experimental task included a practice set of items, which was presented for the first $20 \mathrm{sec}$ of the run. This practice set was included in order to reacquaint the subjects 
with the task and to allow time for steady state magnetization to occur. The data from these practice trials were not included in either the behavioral or the imaging analyses.

Image processing. Functional magnetic resonance images were processed using VoxBo software (www.voxbo.org). Images from the scanner were reconstructed and then sinc interpolated to accommodate the interleaved acquisition sequence. A partial correlation method was applied to each slice in time to identify and remove spatially coherent signal changes due to motion over time (Zarahn, Aguirre, \& D'Esposito, 1997). A six-parameter, rigid-body transformation was used to correct for additional motion (Friston et al., 1995). Only subjects with fewer than $2 \mathrm{~mm}$ of displacement in any direction (plane or angular path) were included in the analysis. This criterion resulted in the exclusion of 1 subject. No spatial smoothing or normalization was performed.

Image analysis. A general linear model for serially correlated error terms (Worsley \& Friston, 1995) was applied to each subject's individual data. Also included in the model was an estimate of intrinsic serial autocorrelation (Zarahn et al., 1997). Region-of-interest analyses were performed within the hypothesis-specified regions on individual, unnormalized subject brains. The regions of interest were defined by drawing masks onto individual subjects' anatomical images (see the Appendix for the specific criteria used and Figure 2 for a diagram of the regions of interest on an individual subject's brain). For each subject, a mean time series was calculated by averaging over all of the voxels in the region of interest. Contrasts between experimental conditions and the baseline condition were performed for all voxels for the average time series within each region of interest. In addition, direct contrasts between experimental conditions were performed for all voxels within each region of interest. The average time series across the voxels within each region of interest was used to calculate a variance-normalized effect size measure for each contrast. The effect sizes of the 7 subjects were used as the dependent variable in a random effects analysis.

In addition to the region-of-interest approach described above, a second region-of-interest approach was applied in ventral stream locations on the basis of coordinates provided by previous research on visual attribute retrieval. For all the analyses in which coordinates were used to define a region or to describe the location of activity, the data were first normalized into a standard space.

\section{RESULTS}

\section{Behavioral Data}

For each of the 7 subjects, accuracy (relative to responses given by $80 \%$ of the subjects in the norming sample) and mean response time for correct trials were calculated for each of the four trials (see Table 1). Response time for correct response trials did not significantly differ across experimental conditions $[F(2,12)=0.19$, $p<.82$ ]. However, mean response time for the lexical decision baseline task differed significantly from that for the corresponding experimental task within each condition (see Table 1). The mean response time for the lexical decision task did not differ significantly across the blocked conditions [e.g., lexical decision within the color condition vs. lexical decision within the shape condition; $F(2,12)=0.84, p=.45]$. There were no significant differences in accuracy for the color, shape, and size

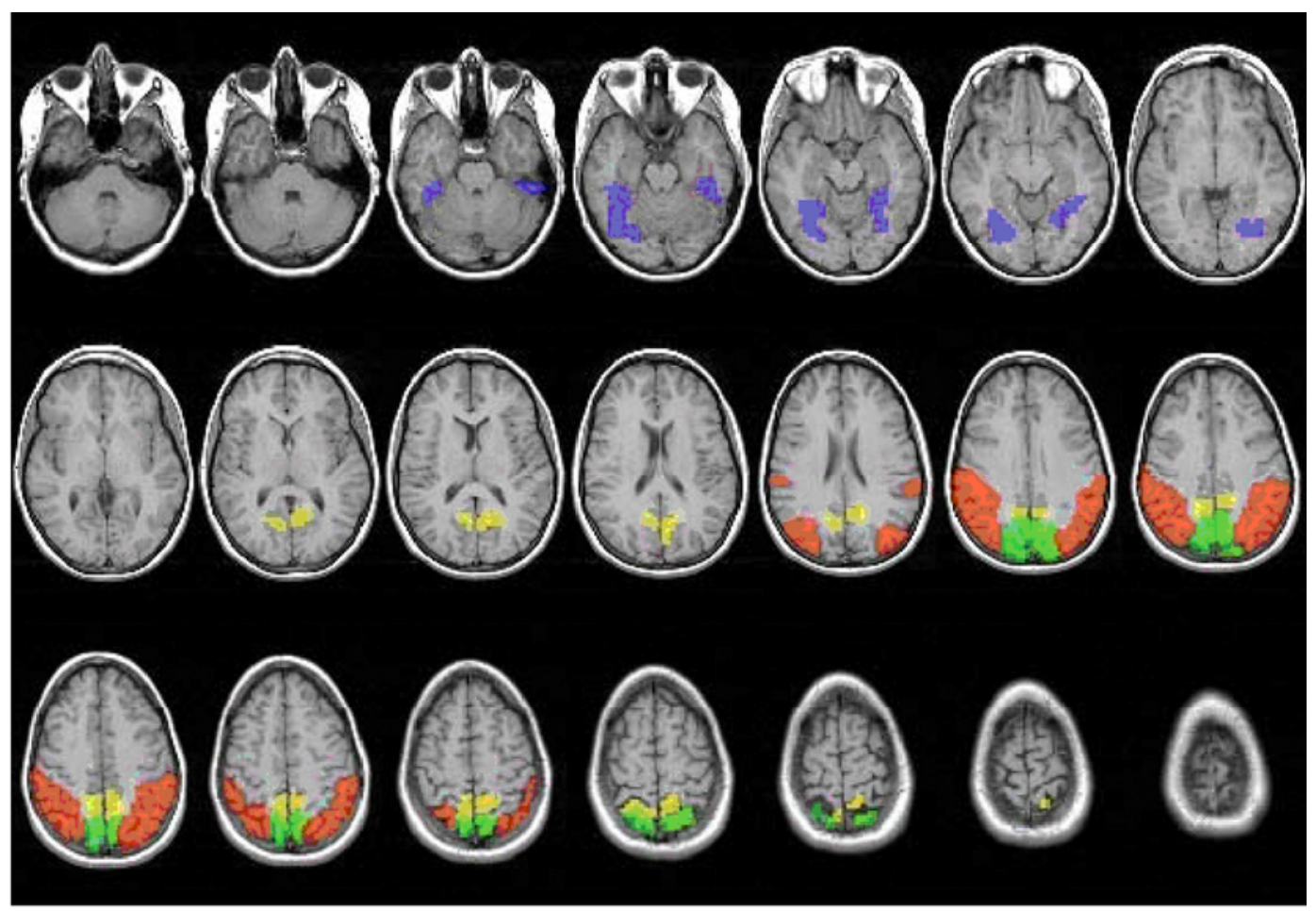

R L

Figure 2. Anatomical masks for each region of interest as seen in one of the 7 subjects. Blue, fusiform gyrus; orange, inferior parietal lobule; green, superior parietal lobule; yellow, precuneus. 
Table 1

Average Response Times (RTs, in Milliseconds), Accuracy (in Percentage Correct) for Each Experimental Condition and for the Nonsemantic Baseline Corresponding to Each Experimental Condition, and $t$ Values for Semantic to Nonsemantic Comparisons

\begin{tabular}{|c|c|c|c|c|c|c|c|c|c|c|}
\hline \multirow[b]{2}{*}{ Condition } & \multicolumn{2}{|c|}{$\begin{array}{c}\text { Semantic } \\
\text { RT }\end{array}$} & \multicolumn{2}{|c|}{$\begin{array}{c}\text { Nonsemantic } \\
\text { RT } \\
\end{array}$} & \multirow{2}{*}{$\begin{array}{l}t \text { Values } \\
\text { (RT) }\end{array}$} & \multicolumn{2}{|c|}{$\begin{array}{l}\text { Semantic } \\
\text { Accuracy }\end{array}$} & \multicolumn{2}{|c|}{$\begin{array}{l}\text { Nonsemantic } \\
\text { Accuracy }\end{array}$} & \multirow{2}{*}{$\begin{array}{c}t \text { Values } \\
\text { (Accuracy) }\end{array}$} \\
\hline & $M$ & $\overline{S D}$ & $M$ & $S D$ & & $M$ & $S D$ & $M$ & $S D$ & \\
\hline Color & 1,162 & 77 & 1,087 & 123 & 2.44 & 88.4 & 5 & 90.5 & 5 & 0.54 \\
\hline Shape & 1,145 & 102 & 1,101 & 144 & 3.10 & 89.1 & 4 & 89.8 & 5 & 0.32 \\
\hline Size & 1,146 & 89 & 1,074 & 105 & 3.97 & 91.7 & 3 & 92.1 & 3 & 0.24 \\
\hline
\end{tabular}

conditions $[F(2,12)=1.00, p=.40]$. Accuracy was not significantly different in the baseline lexical decision task for any of these three tasks $[F(2,12)=0.41, p=$ .67]. Also, $t$ tests revealed no significant differences in accuracy between each of the experimental conditions and its corresponding baseline (see Table 1).

\section{Imaging Data}

Anatomically defined regions of interest. There were two types of planned comparisons that were tested within this data set. The first was a comparison of each semantic retrieval task with the baseline in each of the anatomically defined regions. No main effects were found in the fusiform gyrus for any of the three retrieval tasks (all $t \mathrm{~s}<1$ ). Activity was expected in this region for all three of the semantic retrieval conditions. The failure to find activity in this region is likely to have been due to the size of the area tested relative to the size of the expected activity, and this issue is addressed in the Functionally Defined Regions of Interest section. There were no regions in which size retrieval produced significantly more activity than the baseline task. However, retrieval of shape information relative to the baseline task was associated with activity in the inferior parietal lobule bi- laterally and the left superior parietal lobule, and retrieval of color information relative to the baseline task was associated with activity in the left inferior parietal lobule as well. For a full summary of the main effects for each task in each region, see Table 2.

The second of our planned comparisons, a weighted contrast of size retrieval relative to shape and color retrieval, was performed. None of the regions tested was found to be significantly more active for size retrieval than for shape and color retrieval (see Table 2). Analogous weighted contrasts were performed post hoc for each of the other two experimental conditions. No regions in the dorsal or the ventral streams were found to be significantly active for shape retrieval, relative to color and size retrieval. However, a direct comparison of shape and size with the color condition revealed significant effects in two of the dorsal regions tested [right inferior parietal lobule, $t(6)=3.13, p=.02$; left superior parietal lobule, $t(6)=3.04, p=.02$ ]. In addition, the right precuneus showed marginally significant activity for this contrast $[t(6)=2.83, p=.03]$. No significant effects for this contrast were found in either the left or the right fusiform gyrus or in the left inferior parietal lobule (all $t \mathrm{~s}<1.6)$.

Table 2

Test Statistics ( $t$ Ratios) for the Averaged Effect Sizes of the 7 Subjects for Each Contrast at Each Region of Interest: Weighted Contrasts, Direct Contrasts Between Pairs of Experimental Conditions, and the Main Effects for Each Condition Relative to a Baseline Lexical Decision Task for Each Region

\begin{tabular}{|c|c|c|c|c|c|c|c|c|}
\hline Contrast & $\begin{array}{c}\text { Left } \\
\text { Fusiform }\end{array}$ & $\begin{array}{c}\text { Right } \\
\text { Fusiform }\end{array}$ & $\begin{array}{l}\text { Left } \\
\text { IPL }\end{array}$ & $\begin{array}{l}\text { Right } \\
\text { IPL }\end{array}$ & $\begin{array}{l}\text { Left } \\
\text { SPL }\end{array}$ & $\begin{array}{l}\text { Right } \\
\text { SPL }\end{array}$ & $\begin{array}{c}\text { Left } \\
\text { Precuneus }\end{array}$ & $\begin{array}{c}\text { Right } \\
\text { Precuneus }\end{array}$ \\
\hline \multicolumn{9}{|c|}{ Weighted Direct } \\
\hline Size $-($ shape + color $)$ & -0.4 & 0.3 & -2.1 & 0.8 & 1.4 & 1 & 0.1 & 1.1 \\
\hline Shape $-($ size + color $)$ & 0 & 1.2 & 2.1 & 1.1 & 2 & 1.4 & 2.2 & 1.7 \\
\hline Color - (size + shape $)$ & 0.5 & -1.5 & -0.5 & $-3.1 *$ & $-3^{*}$ & -2.3 & -2.4 & -2.8 \\
\hline \multicolumn{9}{|c|}{ Direct } \\
\hline Size - color & -0.5 & 1.1 & -1.2 & 1.9 & 2.5 & 2 & 1.4 & 1.9 \\
\hline Shape - color & -0.4 & 1.6 & 1.6 & 2.4 & 2.9 & 2.1 & 2.8 & $3.5^{*}$ \\
\hline Size - shape & -0.2 & -0.5 & -2.2 & -0.1 & -0.3 & -0.3 & -1.1 & 0 \\
\hline \multicolumn{9}{|c|}{ Main Effects (Attribute Retrieval - Lexical Decision) } \\
\hline Size - baseline & 0.8 & -0.1 & 1.9 & 2.2 & 1.6 & 0.8 & 0.1 & 0.6 \\
\hline Shape - baseline & 0.7 & 0.6 & $8.5^{*}$ & $2.8 *$ & $3.8^{*}$ & 1.3 & 1.7 & 0.8 \\
\hline Color - baseline & 0.8 & -1.2 & $2.8^{*}$ & 0.2 & -0.5 & -0.7 & -1.2 & -1.8 \\
\hline
\end{tabular}

Note-IPL, inferior parietal lobule; SPL, superior parietal lobule. *Significant at the $p<.05$ level for the main effects and the planned contrast of size - (shape + color), at the $p<.025$ level for the other two weighted direct contrasts [i.e., shape $-($ size + color $)$ and color $-($ size + shape $)$, and at the $p<.0166$ level for the direct contrasts. 
In addition, post hoc direct contrasts were performed in each region between each pairwise combination of conditions, and the resulting effect sizes were averaged across subjects and tested against a null hypothesis of no activation. The effects resulting from these contrasts were as follows: Shape showed significantly greater activity than did color in the right precuneus $[t(6)=3.48$, $p=.013]$ and marginally greater activity in the left superior parietal lobule $[t(6)=2.92, p=.027]$ and the left precuneus $[t(6)=2.78, p=.032]$.

Functionally defined regions of interest. As was suggested above, it may have been the case that the anatomical regions of interest employed in the fusiform gyrus analysis above were too large to detect activity associated with visual attribute retrieval. Given that many other studies have shown activity in the ventral stream associated with visual attribute retrieval, there is reason to depart from the anatomical approach that was adopted for the exploratory analysis of the dorsal visual-processing stream. Therefore, a second region-of-interest approach was applied to the fusiform gyrus. In order to apply a functionally defined region-of-interest approach, our images were normalized, and coordinates were selected from previous research studies in which color (e.g., Chao $\&$ Martin, 1999) and size (e.g., Rossion et al., 2000) retrieval were tested separately. Because there were no studies in which shape retrieval was specifically tested, the coordinate selected to test for activity associated with our shape retrieval task was drawn from a study in which shape perception was tested for (e.g., Martin, Wiggs, Ungerleider, \& Haxby, 1996). The Talairach coordinates (Talairach \& Tournoux, 1988) for local maxima of activity in the ventral stream for each of the selected research studies were used as the center of a 27-voxel spherical region tested in our present data set.

We would have preferred to use Kellenbach et al.'s (2001) local maxima for size retrieval, because theirs was the most recent study of size knowledge retrieval, but the local maxima that they obtained in their PET study were outside our data collection range. Specifically, the local maxima that they reported were lower in the $z$ dimension than our data coverage range. Instead, we formed a spherical region of interest around a coordinate reported by Rossion et al. (2000) for a size retrieval task. Rossion et al.'s size task required subjects to decide which of two visually presented animals was the larger or smaller one in its real size (the animals were presented out of context, and not in consistent size relationship with the paired animal). Our size task was found to activate the portion of the left fusiform gyrus $(-42,-48$, $-14)$ that was defined on the basis of Rossion et al.'s local maxima for size knowledge retrieval $[t(6)=2.82$, $p=.02]$. Of the three fusiform gyrus coordinates that Rossion et al. reported to be associated with size retrieval, this one was chosen as the closest in the $z$ dimension to the coordinate reported by Kellenbach et al. (2001) for size retrieval, relative to baseline.

Since most studies of visual knowledge retrieval have not singled out shape retrieval from other types of visual attribute retrieval, a shape perception task (Martin et al., 1996) was used to define the functional region of interest to test for shape activity in our data set. Martin et al. (1996) compared activity associated with viewing nonsense objects relative to viewing visual noise. Martin et al. (1996) proposed that the response of this ventral stream region to nonsense objects may indicate its involvement in shape processing during perception. In the present study, we found no significant activity for our shape retrieval task in the regions of interest defined using the coordinates from Martin et al.'s study [left inferior occipital/fusiform gyrus, $-38,-82,-4 ; t(6)=$ $0.42, p=.35$; right inferior occipital/fusiform gyrus, 34 , $-72,-8 ; t(6)=0.15, p=.44]$. However, multiple studies of semantic memory retrieval have shown activity that is $1-2 \mathrm{~cm}$ anterior to sensorimotor regions (for a review, see Thompson-Schill, 2003). It is possible that retrieval of form information from memory involves brain regions that are also shifted relative to the regions involved in form perception.

Color knowledge retrieval is the most extensively studied out of the three visual attributes that were investigated in our study. The coordinates we selected to define the functional region of interest for color knowledge retrieval were gathered from a portion of Chao and Martin's (1999) PET study, in which colors were generated for objects presented in grayscale. Chao and Martin's study on color retrieval was chosen because Martin et al. (1996) tested semantic retrieval of color information on multiple occasions and this particular study included a comprehensive test of color knowledge. When our color retrieval task was compared with the baseline task in the sphere formed around the coordinates of Chao and Martin's local maxima in each hemisphere, a trend was observed in the left fusiform gyrus [BA 18: $-38,-76,-8$ in Talairach space; $t(6)=1.43, p=.10$ ] but not in the right [BA 19: 38, $-64,-16 ; t(6)=-1.75, p=.93$ ].

\section{Whole-Brain Analysis}

A whole-brain analysis was performed to provide readers with a visual reference for the activity observed across subjects. $T$ maps of each of the main effects at a threshold of $t>6.5, p<.0006$, uncorrected, can be viewed in Figures 3-5. Also, Tables 3-5 provide the coordinates of local maxima at a threshold of 6.5. None of the whole-brain analyses revealed significant activity at a Bonferroni corrected threshold of $t(6)=16.96$.

\section{DISCUSSION}

Previous investigators of the neural basis of visual knowledge have focused on the role of the ventral visual stream (e.g., the fusiform gyrus) in conceptual retrieval. In this study, we examined whether retrieval of different types of visual attributes-namely, color, shape, and size-would differentially involve the dorsal visualprocessing stream. Consistent with domain-specific distributed models of semantic memory (e.g., Allport, 1985), retrieval of different types of visual attribute knowledge 


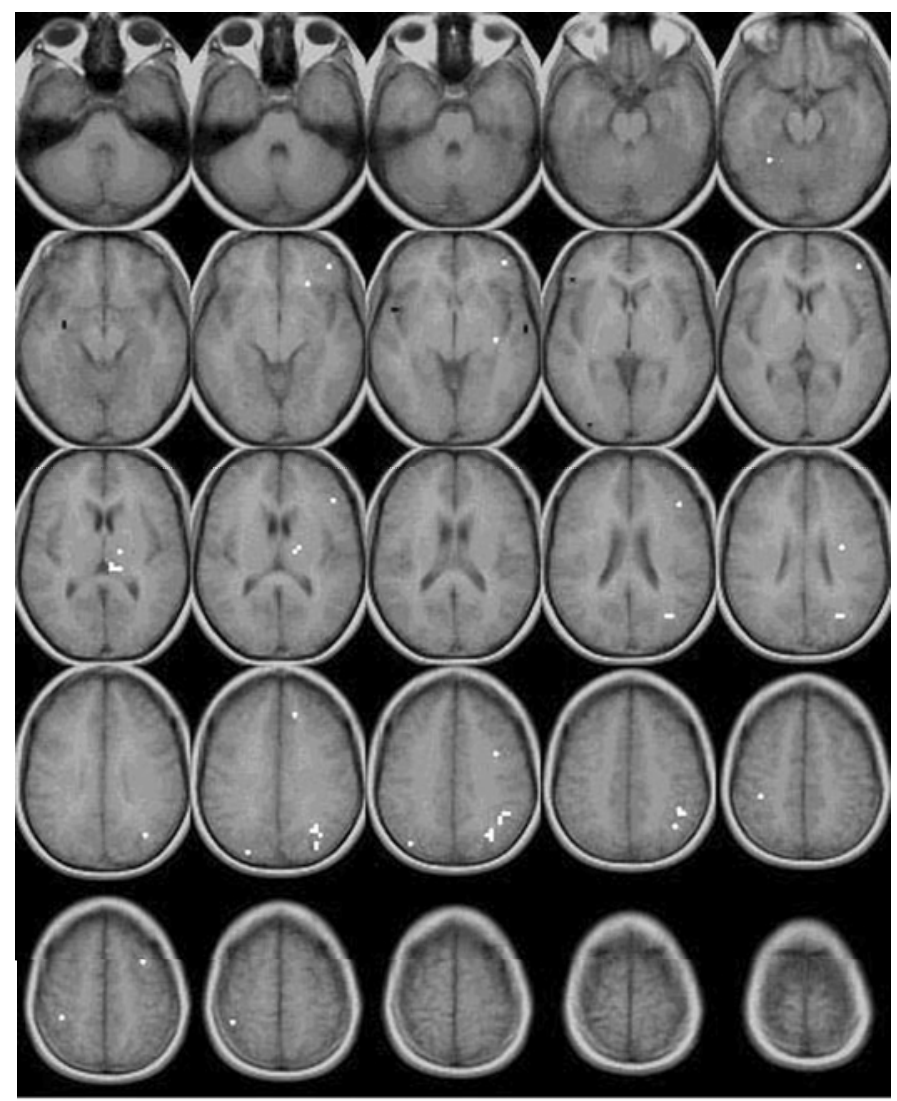

R L

Figure 3. Shape retrieval $>$ baseline thresholded at $t>6.5$. White, activation; black, deactivation.

were associated with different sensorimotor areas. Specifically, in this study, we reported differences in the involvement of the posterior parietal cortex during retrieval of shape and size knowledge, relative to retrieval of color knowledge. We found left superior parietal lobule and right inferior parietal lobule activity for the weighted comparison of size and shape retrieval with color retrieval. Furthermore, direct contrasts of shape and color retrieval revealed that shape retrieval was associated with significant activation of the right precuneus.

In a similar study, Kellenbach et al. (2001) proposed that spatial processing may be necessary for the acquisition of knowledge about the size of objects (e.g., determination of location in order to infer actual size, comparison with other objects in the environment). Domain-specific models of semantic memory (e.g., Allport, 1985) describe distributed networks of conceptual knowledge that are linked to the sensorimotor areas involved in perception of those attributes. Following these accounts, one would predict that cortical areas involved in spatial processing would also be active during retrieval of knowledge about object size. In line with their hypothesis, Kellenbach et al. found greater activity for size re- trieval than for color retrieval in the precuneus, a region within the dorsal visual-processing stream. In contrast, they reported color retrieval to be associated with ventral, inferior temporal lobe activity, but not with posterior parietal activity. The authors acknowledged that medial dorsal areas, such as the precuneus, are less commonly associated with spatial processing than are lateral parietal regions. Nonetheless, the general hypothesis that retrieval of size information involves dorsal stream processing that is not common to shape and color information is supported by some of the neuropsychological literature. Specifically, Coltheart et al. (1998) have reported a patient who had spared size knowledge with deficits for other visual attributes, such as shape and color.

On the basis of the results of Kellenbach et al.'s (2001) study, we expected that size would activate a region within the dorsal stream, whereas color would not. This hypothesis is also consistent with proposals made in both the semantic memory literature and the mental imagery literature. In their review of the literature on mental imagery, Kosslyn and Thompson (2000) drew a distinction between spatial imagery and figural imagery: Spatial imagery focuses on spatial relations, whereas figural im- 


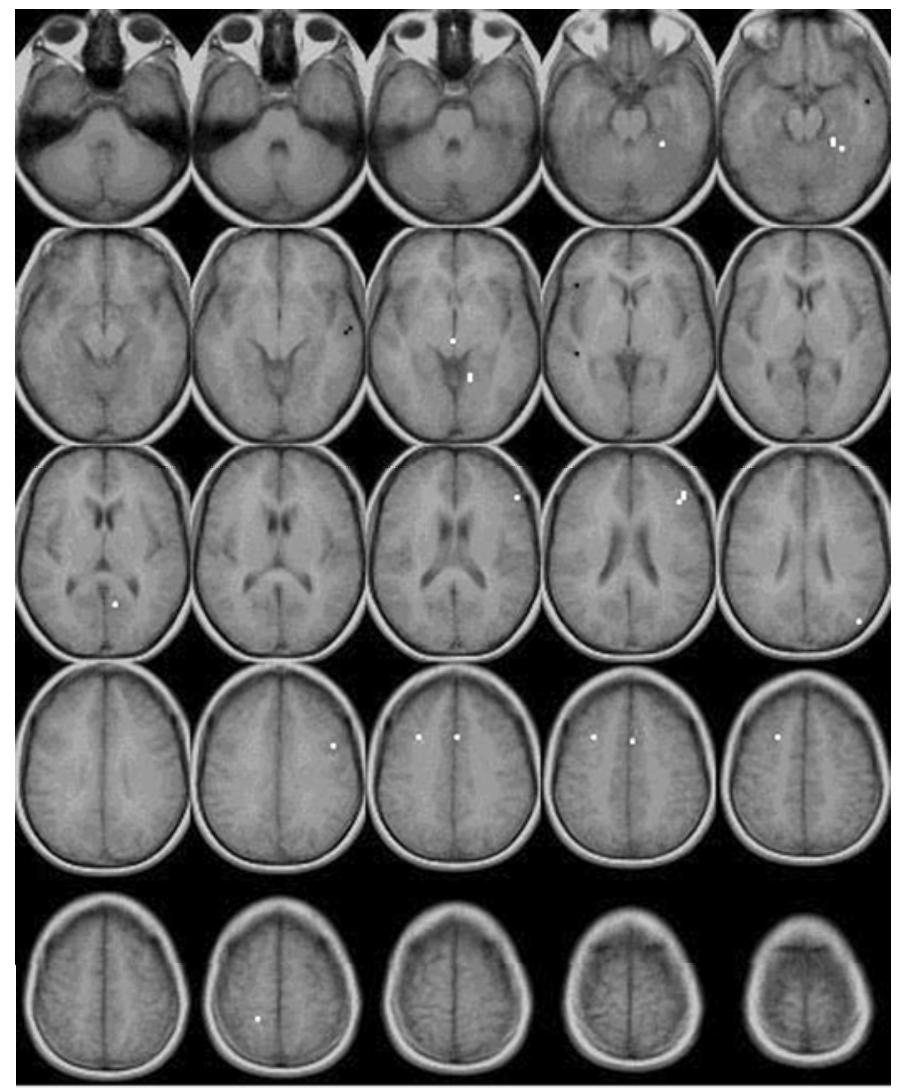

R L

Figure 4. Size $>$ baseline thresholded at $t>6.5$. White, activation; black, deactivation.

agery involves retrieval of shape and other object features to form a low-resolution image. They related spatial imagery to the posterior parietal lobe and figural imagery to the posterior, inferior temporal lobe. Therefore, the semantic memory literature and the mental imagery literature both draw a division between dorsal and ventral stream processing and relate retrieval of spatial information to the dorsal stream.

Retrieval of size knowledge did activate regions of the dorsal visual-processing stream, as predicted by Kellenbach et al. (2001). However, the inclusion of a shape retrieval task in the present design suggests a somewhat different interpretation of the results.

The novel finding in the present study was that shape retrieval activated the posterior parietal cortex. Shape retrieval has generally been suggested to be associated with ventral stream activity in both the semantic memory literature and the mental imagery literature (Ishai, Ungerleider, Martin, \& Haxby, 2000; Ishai, Ungerleider, Martin, Schouten, \& Haxby, 1999; Kosslyn \& Thompson, 2000). Indeed, Lambert, Sampaio, Scheiber, and Mauss's (2002) animal imagery study is the only mental imagery study to our knowledge that has reported dorsal stream activity associated with imagery in a nonspatial task. Other mental imagery research has found posterior parietal activity only in shape tasks that were particularly spatial in nature (e.g., Mellet et al., 1996). However, visual perception research in animals (Sakata et al., 1997; Sereno, Trinath, Augath, \& Logothetis, 2002) and humans (Faillenot, Toni, Decety, Gregoire, \& Jeannerod, 1997; Kourtzi \& Kanwisher, 2000; Kraut, Hart, Soher, \& Gordon, 1997) has suggested a role for the dorsal stream in the processing of shape information. Furthermore, some neuropsychological research is consistent with a functional dissociation of color and other visual attributes. For example, Miceli et al. (2001) have reported a patient who had impaired knowledge of object color as a result of a stroke. Despite her loss of color knowledge, her knowledge of object form, size, and function was reported to be intact. Various explanations for the finding of dorsal stream activity during shape retrieval will be proposed below.

On the basis of previous studies of semantic retrieval of visual information (Kellenbach et al., 2001; Martin et al., 1995; Thompson-Schill et al., 1999; Vandenberghe et al., 1996), it was expected that the shape, color, and size retrieval tasks would activate the ventral temporal lobe. In the absence of any neuroimaging studies 


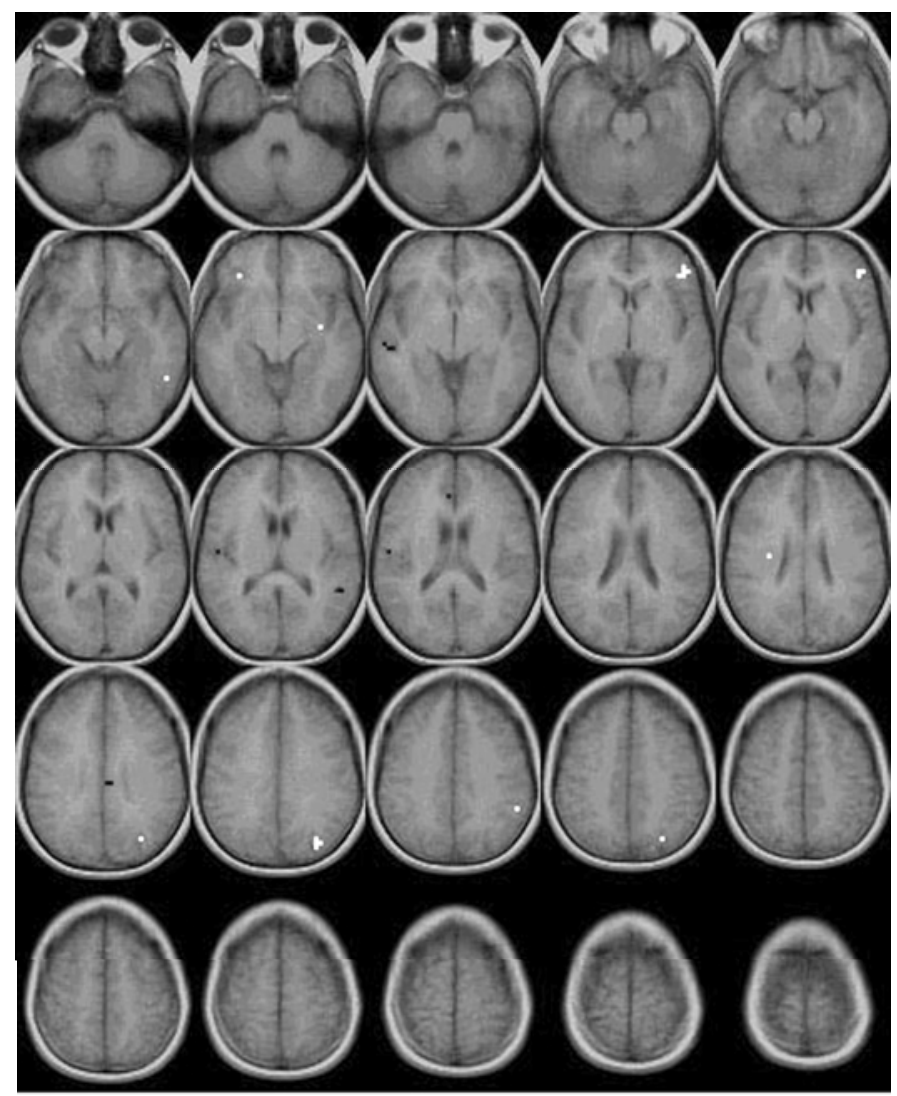

R L

Figure 5. Color retrieval $>$ baseline thresholded at $t>6.5$. White, activation; black, deactivation.

testing semantic retrieval of shape information specifically, ventral temporal activity was hypothesized on the basis of results from general visual attribute retrieval (e.g., Thompson-Schill et al., 1999) and from studies of mental imagery (e.g., D'Esposito et al., 1997; Lambert et al., 2002). A significant effect for size retrieval and a marginally significant effect for color retrieval was found in the fusiform gyrus when the images were normalized and regions of interest were formed around coordinates drawn from previous literature.

\section{Interpretations of Dorsal Stream Activity Associated With Shape Retrieval}

The central finding in this study was the observation of greater activation for size and shape versus color retrieval in the dorsal stream. There are a number of interpretations of this result that we will consider here. One class of interpretations has to do with the acquisition of knowledge about size and shape and the reasons acquisition of this knowledge involves the posterior parietal cortex, whereas acquisition of color knowledge does not. The dorsal stream is preferentially involved in the processing of certain kinds of visual information about objects; therefore, it should be preferentially involved in the retrieval of this kind of information, according to distributed models of semantic memory (e.g., Allport, 1985). The specific pattern of greater dorsal activity during shape and size versus color retrieval might indicate dorsal stream involvement in the processing of shape and size information, either because acquisition of this information involves spatial processors or because it involves grasping or tactile interaction. Each of these hypotheses will be discussed in more detail below. In a second class of interpretations, the possibility that the observed parietal activation has less to do with the representations of size and shape than with the processing demands present in this particular experiment is considered. That is, the dorsal stream might have been activated in our experiment because of specific requirements of our tasks that might not be present in all shape and size retrieval tasks. We will consider both kinds of explanations in turn.

One interpretation of this result is that the perception of shape and size both involve spatial processing and, as a consequence, retrieval of shape and size knowledge activates dorsal visual stream regions involved in spatial processing (i.e., the "where" pathway; Ungerleider \& Mishkin, 1982). As was mentioned earlier, size retrieval 
Table 3

Local Maxima for Shape Retrieval - Baseline

\begin{tabular}{|c|c|c|c|c|c|c|c|}
\hline \multirow[b]{2}{*}{ Region } & \multirow[b]{2}{*}{ Hemisphere } & \multicolumn{3}{|c|}{ Coordinate } & \multirow[b]{2}{*}{$T$} & \multirow[b]{2}{*}{ BA } & \multirow[b]{2}{*}{ Structure } \\
\hline & & $x$ & $y$ & $z$ & & & \\
\hline \multirow[t]{7}{*}{ Frontal lobe } & Left & -48 & 40 & -6 & 7.58 & 47 & inferior frontal gyrus \\
\hline & & -48 & 26 & 3 & 7.22 & 45 & inferior frontal gyrus \\
\hline & & -45 & 22 & 13 & 11.93 & 45 & inferior frontal gyrus \\
\hline & & -15 & 30 & 26 & 7.12 & 32 & medial frontal gyrus \\
\hline & & -37 & -2 & 32 & 6.54 & 6 & precentral gyrus \\
\hline & & -22 & 25 & -18 & 7.92 & 11 & middle frontal gyrus \\
\hline & & -45 & 43 & -15 & 6.8 & 12 & middle frontal gyrus \\
\hline \multirow[t]{2}{*}{ Temporal lobe } & Left & -33 & 2 & -34 & 11.2 & 21 & middle temporal gyrus \\
\hline & & -37 & -23 & -15 & 8.32 & 36 & parahippocampal gyrus \\
\hline \multirow[t]{2}{*}{ Occipital lobe } & Left & -33 & -82 & 32 & 8.34 & 19 & superior occipital gyrus \\
\hline & Right & 26 & -86 & 32 & 7.36 & 19 & cuneus \\
\hline \multirow[t]{5}{*}{ Parietal lobe } & Left & -41 & -59 & 40 & 8.16 & 40 & inferior parietal lobule \\
\hline & & -30 & -68 & 31 & 15.89 & 19 & precuneus \\
\hline & & -41 & -57 & 35 & 13.76 & 39 & angular gyrus \\
\hline & Right & 37 & -78 & 36 & 8.13 & 19 & precuneus \\
\hline & & 41 & -45 & 52 & 7.93 & 40 & inferior parietal lobule \\
\hline \multirow[t]{4}{*}{ Subcortical } & Left & -33 & -17 & 19 & 6.88 & 13 & insula \\
\hline & & -11 & -33 & 2 & 8.65 & & thalamus \\
\hline & & -15 & -18 & 6 & 12.21 & & thalamus \\
\hline & Right & 30 & -56 & -22 & 6.58 & & right cerebellum \\
\hline
\end{tabular}

Note-The threshold to maintain a mapwise $\propto=.05$ for significance is $t>16.96$.

has been thought to involve spatial processors, due to the spatial comparisons made between objects during size discrimination (Kellenbach et al., 2001). Even if objects need not be compared against one another to glean shape information, there still may be spatial comparisons at work during shape discrimination. Objects may be spatially processed as the parts and angles within the object are integrated into a united shape, separate from the background. Neither between-object nor within-object spatial comparisons seem likely to be utilized during color perception. Therefore, under this explanation, size retrieval involves spatial comparisons between objects, shape retrieval involves spatial comparisons within objects, and color retrieval involves no spatial comparisons whatsoever. However, arguing against this explanation is the fact that most patients with spatial-processing impairments are not impaired in identifying objects (Jeannerod, Decety, \& Michel, 1994; Rizzo \& Vecera, 2002).

Another possible explanation of this result is that perception of object shape and size is informed, in part, by sensorimotor interactions and, thus, retrieval of shape and size knowledge activates dorsal visual stream regions involved in visually mediated actions (e.g., the "how" pathway; Goodale \& Milner, 1992). In other words, one source of knowledge of object shape and size is information acquired by the grasping of objects: When a person reaches to grasp an object, he or she must estimate the absolute size and the shape of the object. As he or she comes closer to the object, he or she may reassess the size and shape of the object and so learn about these attributes during the reaching and grasping process. If size and shape information can be learned in this way, distributed models of semantic memory would predict that part of the representation of size and shape information should be in the dorsal stream, with the areas mediating grasping. In contrast, no new information can be learned about the color of an object by attempting to grasp the object. Therefore, one would not expect color to have a representation in the dorsal stream. ${ }^{1}$

The role of the parietal lobe in visually mediated grasping has been well documented in electrophysiological studies with nonhuman primates (see Creem \& Proffitt, 2001, for an in-depth review of the human and primate literature on dorsal stream function). Murata, Sakata, and colleagues have reported form-selective neurons in the anterior intraparietal sulcus (AIP) of monkeys trained to grasp objects of various shapes (Murata, Gallese, Kaseda, \& Sakata, 1996; Murata, Gallese, Luppino, Kaseda, \& Sakata, 2000; Sakata, Taira, Murata, \& Mine, 1995; Taira, Mine, Georgopoulos, Murata, \& Sakata, 1990). Murata et al. (2000) described two different types of shape-selective neurons in the AIP. One type responds most strongly to a preferred object form whenever it is visually presented, regardless of whether the object is actually being grasped. The second type also responds most strongly when its preferred object is visible but increases its firing rate during grasping of the preferred stimulus. These neuron types seem to code information about the form of objects. In addition, they reported other neurons in the AIP that responded only at the moment its preferred object was grasped, regardless of whether the object was in view (light) or not in view (dark). These neurons may code for the particular hand postures associated with gripping particular objects. The results of this study and others by this group (Sakata et al., 1997) suggest that the AIP is involved with threedimensional shape perception, in addition to the inferotemporal cortex, as reported by Janssen, Vogels, and Orban (2000). Sakata et al. (1995) proposed that the visual neurons in the AIP serve to send visual/spatial in- 
Table 4

Local Maxima for Size Retrieval - Baseline

\begin{tabular}{|c|c|c|c|c|c|c|c|}
\hline \multirow[b]{2}{*}{ Region } & \multirow[b]{2}{*}{ Hemisphere } & \multicolumn{3}{|c|}{ Coordinate } & \multirow[b]{2}{*}{$T$} & \multirow[b]{2}{*}{ BA } & \multirow[b]{2}{*}{ Structure } \\
\hline & & $x$ & $y$ & $z$ & & & \\
\hline \multirow{4}{*}{ Frontal lobe } & Left & -52 & 26 & 8 & 7.03 & 45 & inferior frontal gyrus \\
\hline & & -48 & 26 & 13 & 7.68 & 46 & inferior frontal gyrus \\
\hline & & -48 & 1 & 28 & 7.78 & 6 & precentral gyrus \\
\hline & Right & 33 & 9 & 36 & 9.22 & 9 & precentral gyrus \\
\hline \multirow[t]{4}{*}{ Temporal lobe } & Left & -26 & -41 & -23 & 10.69 & 20 & fusiform gyrus \\
\hline & & -45 & -79 & 22 & 11.24 & 39 & middle temporal gyrus \\
\hline & & -4 & 5 & 37 & 7.35 & 24 & cingulate gyrus \\
\hline & Right & 22 & 9 & 41 & 7.17 & 32 & cingulate gyrus \\
\hline \multirow{2}{*}{ Occipital lobe } & Left & -15 & -63 & -9 & 9.72 & 19 & lingual gyrus \\
\hline & & -11 & -69 & 3 & 9.27 & 18 & lingual gyrus \\
\hline Parietal lobe & Right & 19 & -45 & 53 & 8.31 & 7 & precuneus \\
\hline Subcortical & Left & 0 & -26 & -11 & 7.08 & & red nucleus \\
\hline
\end{tabular}

Note-The threshold to maintain a mapwise $\propto=.05$ for significance is $t>16.96$.

formation on to the premotor cortex to elicit a motor plan for grasping the object. The premotor cortex may then send feedback signals to the AIP that allow it to compare the visual and motor information about the object and the planned grasp. The AIP then returns signals to the premotor cortex that either facilitate the plan of action or inhibit the original plan, so that signals to adjust the hand shape or maintain its posture are conveyed from the premotor cortex to the primary motor cortex.

Contributing to this body of knowledge on the relationship of the AIP area to grasping, Gallese, Murata, Kaseda, Niki, and Sakata (1994) reported that inhibiting activity in the AIP by way of an injected GABA agonist served to impair monkeys' ability to shape the hand appropriately to grasp objects. In their review of the literature on parietal lobe function, Culham and Kanwisher (2001) noted that the monkey AIP probably corresponds to the AIP in humans, and this area has also been implicated in human grasping behavior, on the basis of neuroimaging evidence (Binkofski et al., 1998; Faillenot et al., 1997). In addition, Buxbaum and Saffran (2002) have reported that nine patients with left inferior parietal damage were impaired in their ability to recognize and form the appropriate grasp shape for familiar objects but were able to form the appropriate grasp shape for novel objects presented to them. This finding suggests that patients with damage to this region have suffered damage to their ability to recall the hand postures that go with familiar objects. In light of these findings, the result of the present study - that retrieval of shape and size information activates the dorsal visual-processing stream-is also in line with the predictions of domain-specific distributed models. Jeannerod (1997) makes a similar proposal regarding storage of visual information, although he suggests that the representation of information in the dorsal stream is pragmatic, rather than semantic.

A related interpretation of the central finding of this study is based on differences in the modality of acquisition of each type of attribute. Size and shape information can be learned through tactile interaction, in addition to visual interaction. Thus, the additional activity found in the dorsal stream for size and shape retrieval over color retrieval could reflect the impact of tactile information obtained through the grasping and touching of objects, rather than knowledge acquisition based on the involvement of visual codes that guide grasping. This interpretation would provide further support for domainspecific distributed models under which retrieval of semantic information is thought to involve the regions by which the information was originally acquired.

These three explanations-based on differences in spatial, tactile, and grasping information for shape and size, relative to color perception-all make predictions about differences in the representation of knowledge of

Table 5

Local Maxima for the Contrast Color - Baseline

\begin{tabular}{|c|c|c|c|c|c|c|c|}
\hline \multirow[b]{2}{*}{ Region } & \multirow[b]{2}{*}{ Hemisphere } & \multicolumn{3}{|c|}{ Coordinate } & \multirow[b]{2}{*}{$T$} & \multirow[b]{2}{*}{ BA } & \multirow[b]{2}{*}{ Structure } \\
\hline & & $x$ & $y$ & $z$ & & & \\
\hline \multirow[t]{2}{*}{ Frontal lobe } & Left & -45 & 32 & -10 & 10.58 & 47 & inferior frontal gyrus \\
\hline & Right & 33 & 32 & -18 & 8.38 & 47 & inferior frontal gyrus \\
\hline \multirow[t]{2}{*}{ Temporal lobe } & Left & -37 & -11 & -16 & 7.01 & 20 & sub-gyral \\
\hline & & -56 & -59 & -18 & 7.69 & 37 & fusiform gyrus \\
\hline Occipital lobe & Left & -33 & -78 & 32 & 12.46 & 19 & superior occipital gyrus \\
\hline Parietal lobe & Left & $\begin{array}{l}-56 \\
-30\end{array}$ & $\begin{array}{l}-49 \\
-74\end{array}$ & $\begin{array}{l}35 \\
41\end{array}$ & $\begin{array}{l}7.17 \\
7.86\end{array}$ & $\begin{array}{l}40 \\
19\end{array}$ & $\begin{array}{l}\text { supramarginal gyrus } \\
\text { precuneus }\end{array}$ \\
\hline Subcortical & Right & 30 & -21 & 19 & 9.12 & & claustrum \\
\hline
\end{tabular}

Note-The threshold to maintain a mapwise $\propto=.05$ for significance is $t>16.96$. 
these visual attributes. However, the present study (and perhaps any fMRI study) is unable to distinguish between activity reflecting the location of stored information and activation related to on-line computation of these properties.

Therefore, one possibility that cannot be ruled out is that the present results may have been a consequence of the specific tasks used and, thus, may not indicate representational differences between visual semantic attributes per se. In the shape retrieval task, the subjects were asked to decide whether a named object was "mostly curved or mostly straight." The subjects may have mentally rotated each named object during this task, so as to check the majority of its edges for curvature. Mental rotation is generally associated with activation in parietal regions (Alivisatos \& Petrides, 1997; Podzebenko, Egan, $\&$ Watson, 2002). Because the size and color tasks were not likely to have evoked such processing, a mental rotation explanation for the dorsal activity associated with the shape task is plausible. Under this explanation, size and shape activate the posterior parts of the parietal lobe for different reasons: mental rotation for shape and spatial comparison for size.

A second task-based explanation is that the subjects may have mentally imagined grasping the objects during the size and shape tasks, but not during the color task. In other words, dorsal stream activation might reflect processes that are incidental to the semantic retrieval process, and not something about semantic retrieval per se. In determining the size of objects in the present study, the subjects may have considered the size relationship between each object and his or her body. For example, the subjects might have considered whether or not the object could be held in their hands. Similarly, in order to determine the shape of each object named in the shape task, the subjects might have thought about what the object would feel like in their hands. Therefore, both the shape and the size retrieval tasks may have evoked dorsal processing because the subjects envisioned grasping the named objects. Such a strategy is plausible, given that in both conditions, many of the items were manipulable. This explanation differs from the knowledge acquisition accounts described above, insofar as grasping imagery is utilized on line during the shape and size retrieval tasks but is not necessary for retrieval of this information.

Another possibility is that one property, such as size knowledge, may be stored in the parietal lobes and another, such as shape, recruits these regions during retrieval because retrieval of shape knowledge automatically involves retrieval of size information. This possibility cannot be ruled out, but it is still interesting that retrieval of one type of visual attribute information-shape information, for example-would consistently be associated with retrieval of another type of attribute (e.g., size), but not of other visual attributes, such as color.

In sum, we found dorsal stream activity associated with size and shape retrieval relative to color retrieval. These results are consistent with domain-specific dis- tributed models of semantic memory and suggest that shape information has a representation in the dorsal stream that may be related to spatial processing, tactile processing, or processing of visual information to guide reaching behavior. However, it is possible that these results may relate to the specific task used. These interpretations are not mutually exclusive, and the dorsal stream activity seen in this study could be due to a combination of two or more of the explanations discussed. Future studies will be necessary to address these and, perhaps, other interpretations of the role of the dorsal visual-processing stream in retrieval of knowledge of object appearance. Either way, the results point to interesting differences between retrieval of different aspects of visual knowledge that may inform theories of the role of the dorsal visual-processing stream.

\section{REFERENCES}

Alivisatos, B., \& Petrides, M. (1997). Functional activation of the human brain during mental rotation. Neuropsychologia, 35, 111-118. ALLPORT, D. A. (1985). Distributed memory, modular subsystems and dysphasia. In S. K. Newman \& R. Epstein (Eds.), Current perspectives in dysphasia (pp. 32-60). Edinburgh: Churchill Livingstone.

Binkofski, F., Dohle, C., Posse, S., Stephan, K. M., Hefter, H., SEITZ, R. J., \& FREUND, H. J. (1998). Human anterior intraparietal area subserves prehension: A combined lesion and functional MRI activation study. Neurology, 50, 1253-1259.

BuXbaum, L. J., \& SAFFran, E. M. (2002). Knowledge of object manipulation and object function: Dissociations in apraxic and nonapraxic subjects. Brain \& Language, 82, 179-199.

CHAO, L., \& MARTIN, A. (1999). Cortical regions associated with perceiving, naming, and knowing about colors. Journal of Cognitive Neuroscience, 11, 25-35.

Cohen, J. D., MacWhinney, B., Flatt, M., \& Provost, J. (1993). PsyScope: An interactive graphic system for designing and controlling experiments in the psychology laboratory using Macintosh computers. Behavior Research Methods, Instruments, \& Computers, 25, 257-271.

Coltheart, M., Inglis, L., Cupples, L., Michie, P., Bates, A., \& Budd, B. (1998). A semantic subsystem of visual attributes. Neurocase, 4, 353-370.

Creem, S. H., \& Proffitt, D. R. (2001). Defining the cortical visual systems: "What," "Where," and "How." Acta Psychologica, 107, 4368.

Culham, J. C., \& Kanwisher, N. G. (2001). Neuroimaging of cognitive functions in human parietal cortex. Current Opinion in Neurobiology, 11, 157-163.

D'Esposito, M., Detre, J. A., Aguirre, G. K., Stallcup, M., Alsop, D. C., TiPPET, L. J., \& FARAH, M. J. (1997). A functional MRI study of mental image generation. Neuropsychologia, 35, 725-730.

Faillenot, I., Toni, I., Decety, J., Gre Goire, M. C., \& JeAnnerod, M. (1997). Visual pathways for object-oriented action and object recognition: Functional anatomy with PET. Cerebral Cortex, 7, 77-85.

Friston, K. J., Ashburner, J., Frith, C. D., Poline, J. B., Heather, J. D., \& FrackowiaK, R. S. J. (1995). Spatial registration and normalization of images. Human Brain Mapping, 3, 165-189.

Gallese, V., Murata, A., Kaseda, M., NiKi, N., \& SAKATA, H. (1994). Deficit of hand preshaping after muscimol injection in monkey parietal cortex. NeuroReport, 5, 1525-1529.

GoodAle, M. A., \& Milner, A. D. (1992). Separate visual pathways for perception and action. Trends in Neurosciences, 15, 20-25.

Hu, Y., \& Goodale, M. A. (2000). Grasping after a delay shifts sizescaling from absolute to relative metrics. Journal of Cognitive Neuroscience, 12, 856-868.

Ishai, A., Ungerleider, L. G., Martin, A., \& Haxby, J. V. (2000). The representation of objects in the human occipital and temporal cortex. Journal of Cognitive Neuroscience, 12(Suppl. 2), 35-51. 
Ishai, A., Ungerleider, L. G., Martin, A., Schouten, J. L., \& HaXвY, J. V. (1999). Distributed representation of objects in the human ventral visual pathway. Proceedings of the National Academy of Sciences, 96, 9379-9384.

Janssen, P., Vogels, R., \& Orban, G. A., (2000). Three dimensional shape coding in inferior temporal cortex. Neuron, 27, 385-397.

JEANNEROD, M. (1988). The neural and behavioral organization of goal-directed movements. Oxford: Oxford University Press.

JEANNEROD, M. (1997). The cognitive neuroscience of action. Oxford: Blackwell.

Jeannerod, M., Decety, J., \& Michel, F. (1994). Impairment of grasping movements following a bilateral posterior parietal lesion. Neuropsychologia, 32, 369-380.

Kellenbach, M. L., Brett, M., \& Patterson, K. (2001). Large, colorful, or noisy? Attribute- and modality-specific activations during retrieval of perceptual attribute knowledge. Cognitive, Affective, \& Behavioral Neuroscience, 1, 207-221.

KossLyn, S. M., \& THOMPSON, W. L. (2000). Shared mechanisms in visual imagery and visual perception: Insights from cognitive neuroscience. In M. S. Gazzaniga (Ed.), The new cognitive neurosciences (2nd ed., pp. 975-985). Cambridge, MA: MIT Press.

Kourtzi, Z., \& KANWISHER, N. (2000). Cortical regions involved in perceiving object shape. Journal of Neuroscience, 20, 3310-3318.

Kraut, M., Hart, J., Soher, B. J., \& Gordon, B. (1997). Object shape processing in the visual system evaluated using functional MRI. Neurology, 48, 1416-1420.

LAmbert, S., SAmpaio, E., Scheiber, C., \& Mauss, Y. (2002). Neural substrates of animal mental imagery: Calcarine sulcus and dorsal pathway involvement -an fMRI study. Brain Research, 924, 176-183.

Lee, A. C. H., Graham, K. S., Simons, J. S., Hodges, J. R., Owen, A. M., \& PATTERSON, K. (2002). Regional brain activations differ for semantic features but not categories. NeuroReport, 13, 1497-1501.

LEVINE, D. N., WARACH, J., \& FARAH, M. (1985). Two visual systems in mental imagery: Dissociation of "what" and "where" in imagery disorders due to bilateral posterior cerebral lesions. Neurology, 35, $1010-1018$

Martin, A., Haxby, J. V., Lalonde, F. M., Wiggs, C. L., \& UngerLEIDER, L. G. (1995). Discrete cortical regions associated with knowledge of color and knowledge of action. Science, 270, 102-105.

Martin, A., Wiggs, C. L., Ungerleider,L. G., \& HaXby, J. V. (1996). Neural correlates of category-specific knowledge. Nature, 379, 649652.

Mellet, E., Tzourio, N., Crivello, F., Joliot, M., Denis, M., \& MAZOYER, B. (1996). Functional anatomy of spatial mental imagery generated from verbal instructions. Journal of Neuroscience, 16, 6504-6512.

Miceli, G., Fouch, E., Capasso, R., Shelton, J. R., Tomaiuolo, F., \& CARAMAZzA, A. (2001). The dissociation of color from form and function knowledge. Nature Neuroscience, 4, 662-667.

Murata, A., Gallese, V., Kaseda, M., \& SaKata, H. (1996). Parietal neuron related to memory-guided hand manipulation. Journal of Neurophysiology, 75, 2180-2186.

Murata, A., Gallese, V., Luppino, G., Kaseda, M., \& SaKata, H. (2000). Selectivity for the shape, size and orientation of objects for grasping in neurons of monkey parietal area AIP. Journal of Neurophysiology, 83, 2580-2601.

Ogawa, S., Menon, R. S., Tank, D. W., Kim, S.-G., Merkle, H., EllermanN, J. M., \& UGURBIL, K. (1993). Functional brain mapping by blood oxygenation level-dependent contrast magnetic resonance imaging: A comparison of signal characteristics with a biophysical model. Biophysical Journal, 64, 803-812.

Perenin, M.-T., \& Vighetto, A. (1988). Optic ataxia: A specific disruption in visuomotor mechanisms. I: Different aspects of the deficit in reaching for objects. Brain, 111, 643-674.

Podzebenko, K., Egan, G. F., \& Watson, J. D. G. (2002). Widespread dorsal stream activation during a parametric mental rotation task, revealed with functional magnetic resonance imaging. NeuroImage, 15, 547-558.

Rizzo, M., \& Vecera, S. P. (2002). Psychoanatomical substrates of Balint's syndrome. Journal of Neurology, Neurosurgery \& Psychiatry, 72, 162-178.

Rossion, B., Bodart, J.-M., Pourtois, G., Thioux, M., Bol, A., CosNARd, G., Georges, B., Michel, C., \& De Volder, A. (2000). Functional imaging of visual semantic processing in the human brain. Cortex, 36, 579-591.

Sakata, H., Tiara, M., Murata, A., Gallese, V., Tanaka, Y., ShIKATA, E., \& KusUnOKI, M. (1997). Parietal visual neurons coding three-dimensional characteristics of objects and their relation to hand action. In P. Thier \& H.-O. Karnath (Eds.), Parietal lobe contributions to orientation in $3 D$ space (pp. 237-254). Heidelberg: Springer-Verlag.

SaKata, H., Taira, M., Murata, A., \& Mine, S. (1995). Neural mechanisms of visual guidance of hand action in the parietal cortex of the monkey. Cerebral Cortex, 5, 429-438.

Sereno, M. E., Trinath, T., Augath, M., \& Logothetis, N. K. (2002). Three-dimensional shape representation in monkey cortex. Neuron, 33, 635-652.

Smith, E. E., \& Medin, D. L. (1981). Categories and concepts. Cambridge, MA: Harvard University Press.

Taira, M., Mine, S., Georgopoulos, A. P., Murata, A., \& Sakata, H. (1990). Parietal cortex neurons in the monkey related to the visual guidance of hand movement. Experimental Brain Research, 83, 2936.

TALAIRACH, J., \& TOURNOUX, P. (1988). Co-planar stereotaxic atlas of the human brain. Stuttgart: Thieme.

THOMPSON-SCHILL, S. L. (2003). Neuroimaging studies of semantic memory: Inferring "how" from “where." Neuropsychologia, 41, 280292.

Thompson-Schill, S. L., Aguirre, G. K., D'Esposito, M., \& Farah, M. J. (1999). A neural basis for category and modality specificity of semantic knowledge. Neuropsychologia, 37, 671-676.

UNGERLEIDER, L. G., \& MishKIN, M. (1982). Two cortical visual systems. In D. Ingle, M. Goodale, \& R. Mansfield (Eds.), Analysis of visual behavior (pp. 549-586). Cambridge, MA: MIT Press.

Vandenberghe, R., Price, C., Wise, R., Josephs, O., \& Frackowiak, R. S. J. (1996). Functional anatomy of a common semantic system for words and pictures. Nature, 383, 254-256.

Warrington, E. K., \& Shallice, T. (1984). Category specific semantic impairments. Brain, 107, 829-854.

WILSON, M. (1987). MRC Psycholinguistic Database: Machine usable dictionary (Version 2.00) [Online]. Available: http://www.psy.uwa. edu.au/mrcdatabase/mrc2.html.

WORSLEY, K. J., \& FRISTON, K. J. (1995). Analysis of fMRI time-series revisited-again. NeuroImage, 2, 173-181.

Zarahn, E., Aguirre, G. K., \& D’Esposito, M. (1997). Empirical analyses of BOLD fMRI statistics: I. Spatially unsmoothed data collected under null-hypothesis conditions. NeuroImage, 5, 179-197.

\section{NOTE}

1. Hu and Goodale (2000) argued that when size information and other spatial information are used by the dorsal stream for reaching, it is calculated on line. Due to the ever-changing status of this egocentric spatial information, $\mathrm{Hu}$ and Goodale argued that it would not be useful to store the spatial information in the dorsal stream long term. Rather, they proposed that long-term knowledge of size and other spatial attributes would be stored in the ventral stream. Therefore, the proposal that dorsal activity for shape and size may occur because grasping was utilized to learn this attribute information is an extension of the claims of Goodale and Milner's (1992) proposal. 


\section{APPENDIX \\ Mask Drawing Criteria}

When a border is not specified, the mask extends out to the end of the gray matter so as to include only voxels that are at least $50 \%$ gray matter.

\section{Fusiform Gyrus}

Specifically, the posterior portion of the fusiform gyrus was the first region of interest investigated. The average mask size in the left fusiform was 90 voxels $(S D=34$ voxels $)$. The average mask size in the right fusiform was 98 voxels $(S D=20$ voxels). The anterior boundary used to define this region was either the lateral curvature of the collateral sulcus or a horizontal match to the anterior-most part of the cerebellum as seen in an axial view. The posterior boundary was the posterior portion of the collateral sulcus. This area was bordered medially by the cerebellum and by the collateral sulcus, when present. Laterally, the lateral occipitotemporal sulcus served as a border when present, and more superiorly, the temporo-occipital incisure served as a lateral boundary.

\section{Inferior Parietal Lobule}

The inferior parietal lobule was defined as the areas titled inferior parietal, second parieto-occipital "pli de plassage," the supramarginal gyrus, and the angular gyrus. The average mask size in the left inferior parietal lobule was 392 voxels ( $S D=106$ voxels). The mean mask size in the right inferior parietal lobule was 392 voxels $(S D=37$ voxels). The inferior parietal area was bordered anteriorly by the postcentral gyrus and posteriorly by the posterior segment of the lateral fissure. The second parieto-occipital "pli de plassage" was bordered anteriorly by the ascending posterior segment of the superior temporal sulcus and was bordered posteriorly by the intra-occipital sulcus. For both the inferior parietal and the parieto-occipital "pli de plassage," the mask extended medially to the end of the gray matter, encompassing voxels that either were at least $50 \%$ gray matter or were within the borders of gray matter defining the region. Superiorly, the supramarginalgyrus, was included in the mask and was bordered anteriorly by the postcentral sulcus. Also, the superior portion of the inferior parietal lobule mask, the angular gyrus, was included in the mask and was bordered posteriorly by the intraparietal sulcus and the superior parietal gyrus.

\section{Superior Parietal Lobule}

The superior parietal lobule mask included the first parieto-occipital "pli de passage," the superior parietal gyrus (P1), and the bridging lobule across the intraparietalsulcus. The mean size of the left superiorparietal lobule was 221 voxels $(S D=59$ voxels $)$. The mean size of the right superior parietal lobule was 237 voxels $(S D=$ 50 voxels). The first parieto-occipital "pli de passage" was bordered laterally by the intraoccipital sulcus and anteriorly by the parieto-occipital fissure. The superior parietal gyrus (P1) was bordered laterally by the intraparietal sulcus and anteriorly by the precuneus. The bridging lobule across the intraparietal sulcus was also included in the mask and was bordered laterally and medially by the intraparietal sulcus.

\section{Precuneus}

The precuneus mask included only the precuneus. The mean mask size in the left precuneus was 146 voxels $(S D=32$ voxels $)$. The mean mask size in the right precuneus was 140 voxels $(S D=29$ voxels $)$. This inferior part of this region was bordered anteriorly by the cingulated gyrus and posteriorly by the parietooccipital fissure. More superiorly, the precuneus was bordered by the superior parietal gyrus (P1) posteriorly and the cingulated gyrus anteriorly. Further posteriorly still, it was bordered by the subparietal sulcus anteriorly and the superior parietal gyrus posteriorly (P1). In its most superior portions, the precuneus was bordered by the cingulate sulcus. 